\title{
Low temperature high-mobility InZnO thin-film transistors fabricated by excimer laser annealing
}

\author{
Mami Fujii, ${ }^{1,2}$ Yasuaki Ishikawa, ${ }^{1,3}$ Ryoichi Ishihara, ${ }^{4}$ Johan van der Cingel, ${ }^{4}$ \\ Mohammad R. T. Mofrad, ${ }^{4}$ Masahiro Horita,,${ }^{1,3}$ and Yukiharu Uraoka ${ }^{1,3}$ \\ ${ }^{1}$ Nara Institute of Science and Technology, 8916-5 Takayama, Ikoma, Nara 630-0192, Japan \\ ${ }^{2}$ Research Fellowships of the Japan Society for the Promotion of Science, 8 Ichiban-cho, Chiyoda-ku, \\ Tokyo 102-8472, Japan \\ ${ }^{3}$ CREST, Japan Science and Technology Agency, Honcho, Kawaguchi, Saitama 332-0012, Japan \\ ${ }^{4}$ Delft University of Technology, Feldmannweg 17, P.O. Box 5053, 2600 GB Delft, The Netherlands
}

(Received 26 December 2012; accepted 14 March 2013; published online 26 March 2013)

\begin{abstract}
In this study, we successfully achieved a relatively high field-effect mobility of $37.7 \mathrm{~cm}^{2} / \mathrm{Vs}$ in an InZnO thin-film transistor (TFT) fabricated by excimer layer annealing (ELA). The ELA process allowed us to fabricate such a high-performance InZnO TFT at the substrate temperature less than $50^{\circ} \mathrm{C}$ according to thermal calculation. Our analysis revealed that high-energy irradiation in ELA produced a mixed phase of $\mathrm{InZnO}$ and $\mathrm{SiO}_{2}$, leading to the deterioration of TFT characteristics. (C) 2013 American Institute of Physics. [http://dx.doi.org/10.1063/1.4798519]
\end{abstract}

Oxide semiconductor films deposited by sputtering have recently attracted considerable attention in the fields of transparent and flexible electronics for next-generation displays, in comparison with conventional amorphous silicon-based materials. In particular, an $\operatorname{In}_{2} \mathrm{O}_{3}$-doped $\mathrm{ZnO}$ (IZO) thin film is widely recognized as a suitable oxide semiconductor since thin-films transistors (TFTs) with that material in the channel layer yield a higher field-effect mobility than amorphous InGaZnO (a-IGZO) TFTs. ${ }^{1-3}$ In the case of the IZO film, the amorphous phase generally provides a high conductivity of about $400 \Omega^{-1} \mathrm{~cm}^{-1}$, which is much higher than the conductivity suitable for the TFT's channel layer. ${ }^{4,5}$ The conductivity of this material can possibly be reduced by a thermal annealing process. ${ }^{6,7}$ In previous reports, thermal annealing with a relatively high temperature of about $300^{\circ} \mathrm{C}$ has been proposed to produce operative IZO TFTs. ${ }^{89}$ However, such a high-temperature process in postannealing limits the choice for plastic flexible substrates. The excimer laser annealing (ELA) process with short pulses has been widely utilized to achieve a low processing temperature to produce a polycrystalline silicon thin film on a glass substrate. ${ }^{10}$ This technique has also been utilized for oxide-semiconductor materials. Nakata et al. reported the fabrication of a-IGZO TFTs by ELA process, which acted as a postannealing process, and the improvement of the TFTs characteristics by ELA, ${ }^{11}$ suggesting that the ELA process is also a promising technique for improving the characteristics of oxide-semiconductor devices. In this study, we focused on an IZO TFT with a higher field-effect mobility than an a-IGZO TFT. We assumed that an ELA process crystallizes IZO with nanograins easily since this material has a lower crystallization temperature than a-IGZO ${ }^{12,13}$ and that an IZO film composed of nanograins has better characteristics than a noncrystallized IZO TFT since carrier scattering is suppressed in the disordered film. We considered it is necessary to use a laser with a wavelength shorter than $400 \mathrm{~nm}$, for the laser to be absorbed by the IZO film since IZO has a wide band gap (higher than $3 \mathrm{eV}$ ). ${ }^{14}$ We used an $\mathrm{XeCl}$ excimer laser with a sufficiently short wavelength of $308 \mathrm{~nm}$ (photon energy:
$4.02 \mathrm{eV})^{15,16}$ and investigated the effects of the ELA process on the IZO TFT characteristics and film properties.

A thermally oxidized $\mathrm{SiO}_{2}$ film of $100 \mathrm{~nm}$ thickness was formed on a highly doped p-type silicon wafer $(<0.002 \Omega \mathrm{cm})$ as a gate insulator. IZO films with a thickness of $50 \mathrm{~nm}$ were deposited by RF magnetron sputtering on $\mathrm{SiO}_{2} / \mathrm{Si}$ wafers with a gas mixture of argon and oxygen at room temperature. For the introduced oxygen ratio ( $F=$ oxygen flow rate/(oxygen+ argon flow rate) $\times 100 \%$ ) during RF magnetron sputtering, we utilized a condition of $F=10 \%$. Source and drain electrodes, which are 100-nm-thick Mo metal layers, were formed by RF magnetron sputtering and a lift-off process. The fabricated TFT has a channel length $(L)$ of $90 \mu \mathrm{m}$ and a channel width $(W)$ of $10 \mu \mathrm{m}$. The ELA process was carried out by irradiation from the top surface of IZO films, which is a back channel positioned in the TFT structure. The laser conditions used were as follows: a wavelength of $308 \mathrm{~nm}$, one shot with a pulse duration of $25 \mathrm{~ns}$ at full width at half maximum, and a spot size of $2.5 \mathrm{~mm} \times 1.67 \mathrm{~mm}$. The light with a wavelength of $308 \mathrm{~nm}$ penetrates our IZO film at a depth of $48 \mathrm{~nm}$ as shown in Table I, which corresponds to an opportune depth of IZO TFTs with a channel thickness of $50 \mathrm{~nm}$. Therefore, most of the incident beam is absorbed in the entire IZO film. The ELA process was carried out in a nitrogen gas flow chamber at an atmospheric pressure.

After setting the optimal ELA conditions on the basis of the film crystallinity measured by X-ray diffraction (XRD, Rigaku RINT-TTRIII/NM), we evaluated TFT transfer characteristics. In addition, we analyzed the changes in oxygen deficiency and film thicknesses caused by ELA, using X-ray photoelectron spectroscopy (XPS, Shimadzu, KRATOS

TABLE I. Absorption coefficient and penetration length of $\mathrm{InZnO}$ film for the light with a wavelength of $308 \mathrm{~nm}$ measured by spectroscopic ellipsometry.

\begin{tabular}{lc}
\hline Absorption coefficient $\left[\mathrm{cm}^{-1}\right]$ & Penetration length $[\mathrm{nm}]$ \\
\hline $1.74 \times 10^{5}$ & 48.0 \\
\hline \hline
\end{tabular}


AXIS-165) and X-ray refraction (XRR, Rigaku RINT-TTR III/NM) methods.

Some transfer curves of the TFTs with and without ELA are shown in Fig. 1. The ELA was carried out with energy densities of $30 \mathrm{~mJ} / \mathrm{cm}^{2}$ and $97.5 \mathrm{~mJ} / \mathrm{cm}^{2}$. In IZO TFTs without ELA, it was difficult to ensure the OFF region, but the TFT with ELA using the lower energy density of $30 \mathrm{~mJ} / \mathrm{cm}^{2}$ showed a distinguished ON/OFF switching. We achieved a high field-effect mobility $\left(\mu_{\mathrm{FE}}\right)$ of $37.7 \mathrm{~cm}^{2} / \mathrm{Vs}$ and good switching characteristics with a subthreshold swing of $0.18 \mathrm{~V} / \mathrm{dec}$, and an ON/OFF ratio of more than $10^{7}$ in the IZO TFTs, as shown in Fig. 1. However, IZO TFTs with higher-energy-density of $97.5 \mathrm{~mJ} / \mathrm{cm}^{2}$ irradiation exhibited degraded characteristics. We evaluated the crystallinity of IZO films with ELA by XRD measurement (data not shown). The IZO films with ELA with energy densities of 97.5 and $30 \mathrm{~mJ} / \mathrm{cm}^{2}$ showed some peaks that corresponded to the crystal planes of $\operatorname{In}_{2} \mathrm{O}_{3}$, and the maximum crystallite diameter was calculated to be approximately $22 \mathrm{~nm}$ from the full width half maximum for each peak. Note that the intensity of each peak was increased with increasing the laser energy. From the XRD pattern, we also confirmed a broad peak, indicating that the IZO film had a mixture of nanocrystal and amorphous phases. This suggests that ELA is suitable for realizing good uniformity in device characteristics even when a large substrate is used since a channel layer with large grains of micron order size usually deteriorates the yield of device production. It is noted that the IZO film with $30 \mathrm{~mJ} / \mathrm{cm}^{2}$ irradiation also exhibited an XRD pattern showing nanocrystal and amorphous phases although the sharpness of its peaks was much broader than that of the IZO film with $95 \mathrm{~mJ} / \mathrm{cm}^{2}$ irradiation. These results indicate that ELA with the energy density of $97.5 \mathrm{~mJ} / \mathrm{cm}^{2}$ negatively affected the performance of IZO TFTs. In contrast, ELA with the energy density of $30 \mathrm{~mJ} / \mathrm{cm}^{2}$ effectively improved the performances of the IZO TFTs. Although our fabrication conditions have not been optimized yet, we found that this method provided a relatively high $\mu_{\mathrm{FE}}$.

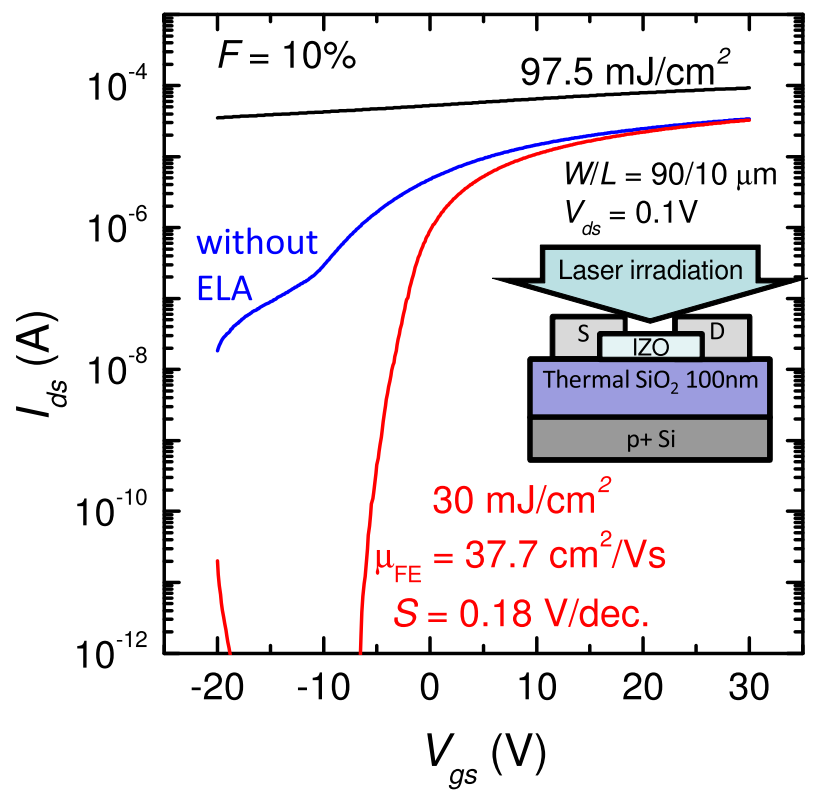

FIG. 1. Transfer curves of IZO TFTs without and with ELA $\left(30 \mathrm{~mJ} / \mathrm{cm}^{2}\right.$ and $97.5 \mathrm{~mJ} / \mathrm{cm}^{2}$ ). The $F$ for IZO film deposition was $10 \%$.
To fabricate a TFT on a plastic substrate, it is necessary to examine the behavior of thermal diffusion in the substrate induced by ELA. We calculated the thermal diffusion from an IZO film surface to a Si substrate surface in a stacked structure of IZO $50 \mathrm{~nm} / \mathrm{SiO}_{2} 100 \mathrm{~nm} / \mathrm{Si}$ substrate using COMSOL Multiphysics engineering simulation software (COMSOL, Inc.). ${ }^{17}$ The optical constants of the IZO film introduced in this calculation were obtained by ellipsometry spectroscopy (M-2000, J. A. Woollam Co., Inc.). The reported physical values of $2 \mathrm{~W} / \mathrm{mK}, 0.39 \mathrm{~J} / \mathrm{gK}$, and $6.7 \mathrm{~g} / \mathrm{cm}^{3}$ were used as the thermal conductivity, specific heat, and mass density of IZO film, respectively. ${ }^{9,18}$ The calculated temperatures at $25 \mathrm{~ns}$ after laser irradiation on the IZO film surface, at the $\mathrm{IZO} / \mathrm{SiO}_{2}$ interface, and on the $\mathrm{Si}$ substrate surface are plotted against laser energy density in Fig. 2. The broken line shows the limit of the stable temperature of the IZO composition. This temperature corresponds with a temperature of sublimation of $\mathrm{In}_{2} \mathrm{O}_{3}$ contained in the IZO film since $\operatorname{In}_{2} \mathrm{O}_{3}$ has a lower sublimation temperature $\left(850^{\circ} \mathrm{C}\right)$ than that of $\mathrm{ZnO}\left(1725^{\circ} \mathrm{C}\right)$. The laser energy density of $97.5 \mathrm{~mJ} / \mathrm{cm}^{2}$ provided a temperature near the $\operatorname{In}_{2} \mathrm{O}_{3}$ sublimation temperature for the IZO surface. However, at the laser energy density of $30 \mathrm{~mJ} / \mathrm{cm}^{2}$, the temperature was calculated to be $216.9^{\circ} \mathrm{C}, 130.7^{\circ} \mathrm{C}$, and $46.9^{\circ} \mathrm{C}$ for the IZO film surface, $\mathrm{IZO} / \mathrm{SiO}_{2}$ interface, and Si substrate surface, respectively, indicating that the temperature became much lower than the sublimation temperature. From this calculation, we found that IZO TFTs with a relatively high $\mu_{F E}$ of approximately $40 \mathrm{~cm}^{2} / \mathrm{Vs}$ can be realized with no apparent thermal damage on the substrate, suggesting that the ELA process provides high-performance TFTs on plastic substrates with a low deformation temperature, such as polyethylene terephthalate.

A discussion on the effect of device performances on an oxygen-deficient region cannot be avoided in the study of devices with oxide semiconductors. Figure 3 shows the $\mathrm{O} 1 \mathrm{~s}$

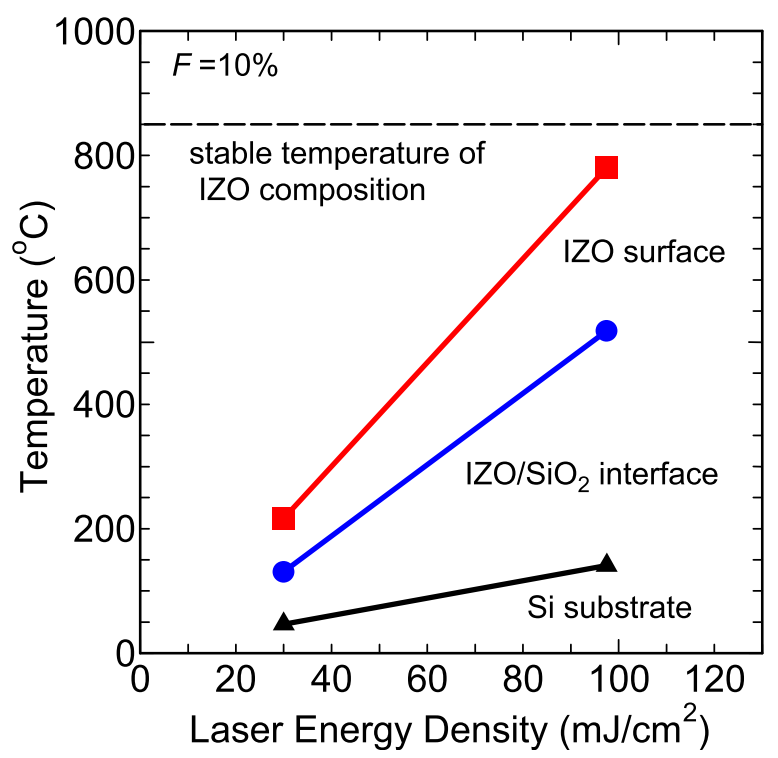

FIG. 2. Temperature calculated using thermal diffusion simulator of IZO/ $\mathrm{SiO}_{2} / \mathrm{Si}$ stacked samples plotted against laser energy density. The square, circle, and triangle show the temperatures on the IZO surface, at the IZO/ $\mathrm{SiO}_{2}$ interface, and on the $\mathrm{Si}$ substrate surface, when IZO was deposited at $F=10 \%$. The broken line indicates the limit of the stable temperature of the IZO film composition. 


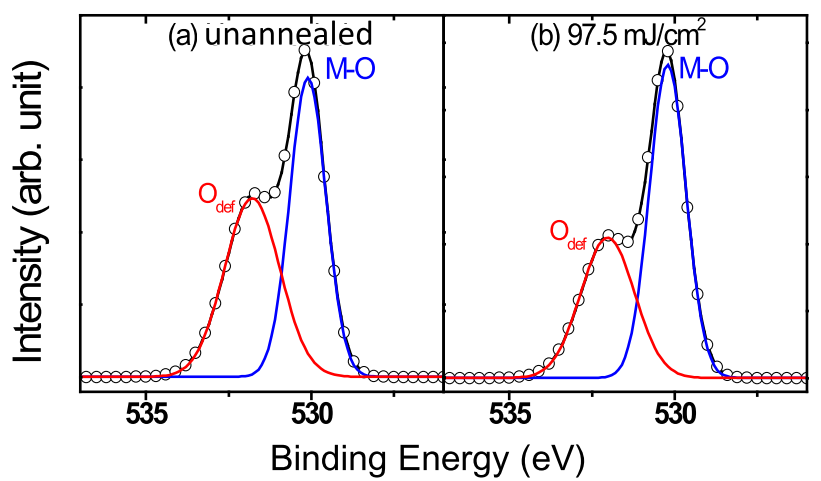

FIG. 3. 1s spectra measured by XPS (a) without ELA and (b) with ELA at (b) $97.5 \mathrm{~mJ} / \mathrm{cm}^{2}$.

spectra measured by XPS (a) without ELA and (b) with laser irradiation at the energy density of $97.5 \mathrm{~mJ} / \mathrm{cm}^{2}$. The main peak was observed at binding energies of $530.2 \pm 0.1 \mathrm{eV}$, and an additional peak was observed at $532.0 \pm 0.2 \mathrm{eV}$. The main and additional peaks correspond to oxygen in oxide lattices without oxygen deficiency (M-O) and with oxygen deficiency $\left(\mathrm{O}_{\mathrm{def}}\right){ }^{19,20}$ The solid line with open circles represents the measured spectrum. The ratios of the $\mathrm{O}_{\text {def }}$ area to the total peak area $\left(\mathrm{O}_{\mathrm{all}}\right)$ are $\mathrm{O}_{\mathrm{def}} / \mathrm{O}_{\mathrm{all}}=47.7 \%$ and $41.3 \%$ in Figs. 3(a) and 3(b), respectively. These figures clearly show that the $\mathrm{O}_{\text {def }}$ peak intensity was reduced by ELA, suggesting that ELA can also reduce the amount of the oxygen deficiency region in the IZO film. It is well known that a low amount of oxygen deficiency leads to the reduction in carrier density, resulting in a low conductivity as an electrical property. However, our results show that a low- $\mathrm{O}_{\mathrm{def}} / \mathrm{O}_{\text {all }}$ film, which was produced with the laser energy density of $97.5 \mathrm{~mJ} / \mathrm{cm}^{2}$, yielded a highly conductive layer and produced poor TFT characteristics. To resolve the discrepancy, we analyzed the structural differences or damage at the $\mathrm{IZO} / \mathrm{SiO}_{2}$ interface caused by ELA in detail. We performed fitting analysis using the XRR spectra of the samples with and without ELA for an $\mathrm{IZO} / \mathrm{SiO}_{2}$ stacked film on a Si substrate. The film thickness calculated by model fitting in XRR is shown in Fig. 4. The stacked film structure used in this fitting was a low-density-IZO/IZO/mixed layer of IZO and $\mathrm{SiO}_{2} / \mathrm{SiO}_{2} / \mathrm{Si}$ as shown on the right side of Fig. 4. The model absenting any layer provided a large fitting error, suggesting the utilized fitting model represents a presumable structure.

The low-density IZO film with a density $<20 \%$ and that of a conventional IZO film showed only moderate changes. This means that the IZO film surface was not damaged by ELA. It is notable that the thicknesses of the $\mathrm{SiO}_{2}$ and IZO films decreased with increasing laser energy owing to the increase in film density. At the same time, an increase in the thickness of the mixed layer of IZO and $\mathrm{SiO}_{2}$ was also observed. Note that the mixed layer did not appear at the energy density of $30 \mathrm{~mJ} / \mathrm{cm}^{2}$. This result implies the diffusion of $\mathrm{Si}$ or $(\mathrm{In}, \mathrm{Zn})$ to the opposite layer, resulting in the formation of the mixed layer of IZO and $\mathrm{SiO}_{2}$. It is known that the IZO film shows a compositional deviation over $300^{\circ} \mathrm{C} .{ }^{12}$ There is no research of observing a Si doping for IZO or In and $\mathrm{Zn}$ doping for $\mathrm{SiO}_{2}$ film using laser irradiation technique, but Aoki et al. and Hatanaka et al. achieved the phosphorous doping to $\mathrm{ZnO}$ layer by excimer laser irradiation with $20 \mathrm{~ns}$

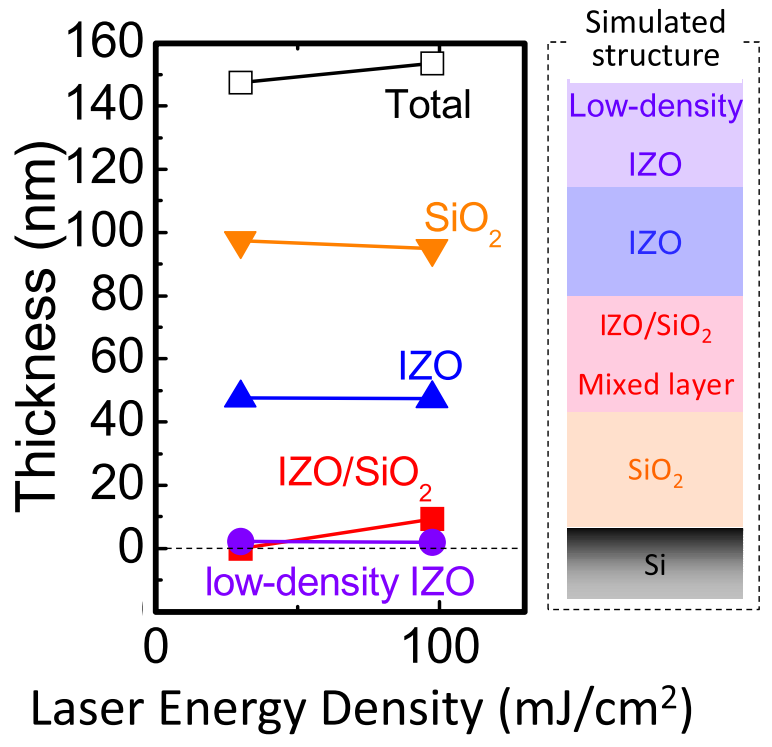

FIG. 4. Film thickness calculated using XRR plotted against laser energy density. The stacked structure used in this model fitting is shown on the right side.

pulse duration time, ${ }^{21,22}$ presuming that the pulse duration time of $25 \mathrm{~ns}$ is enough time for occurrence of a diffusion phenomenon, consequently making the mixed layer. This phenomenon was clearly observed under the irradiation condition of $97.5 \mathrm{~mJ} / \mathrm{cm}^{2}$, which is in good agreement with the thermal simulation results shown in Fig. 2. In addition, the existence of the IZO- $\mathrm{SiO}_{2}$ mixed layer at the interface of the gate insulator considerably affects the electron behavior. Minami et al. reported that $\mathrm{Si}$-doped $\mathrm{ZnO}$ shows a high conductivity. ${ }^{23}$ Although Nakata et al. suggested that the reason of the carrier density increasing in a-IGZO film by irradiation of high energy in ELA process would come from the increasing of the amount of oxygen deficiency, ${ }^{11}$ we obtained lower $\mathrm{O}_{\mathrm{def}}$ by the irradiation of high laser energy of $97.7 \mathrm{~mJ} / \mathrm{cm}^{2}$ compared with the $\mathrm{O}_{\mathrm{def}}$ of no-ELA process, as shown in Fig. 3. Therefore we presume that the high-conductivity mixed layer in the electron mobile region in the channel layer diminished the off state. Owing to this effect, we successfully achieved a high-mobility IZO-TFT with a substrate temperature less than $50^{\circ} \mathrm{C}$ under moderate ELA conditions that do not produce a highly conductive mixed layer.

In conclusion, we discussed the effect of ELA on the characteristics of IZO TFTs to achieve a high-performance TFT with a low-temperature fabrication process. ELA enabled us to fabricate high-mobility IZO TFTs $\left(37.7 \mathrm{~cm}^{2} / \mathrm{Vs}\right)$ without thermal damage to the substrate used. Our results could be attributed to the fact that ELA decreased the amount of oxygen deficiency. The main cause of IZO TFT degradation under the high-laser-energy irradiation of $97.5 \mathrm{~mJ} / \mathrm{cm}^{2}$ was the production of a highly conductive mixed layer of IZO and $\mathrm{SiO}_{2}$.

${ }^{1}$ E. Fortunato, P. Barquinha, A. Pimentel, L. Pereira, G. Goncalves, and R. Martins, Phys. Status Solidi (RRL) 1, R34 (2007).

${ }^{2}$ P.-T. Liu, Y.-T. Chou, and L.-F. Teng, Appl. Phys. Lett. 94, 242101 (2009).

${ }^{3}$ E. Fortunato, P. Barquinha, G. Gonçalves, L. Pereira, and R. Martins, Solid-State Electron. 52, 443 (2008). 
${ }^{4}$ K.-M. Uang, S.-J. Wang, S.-L. Chen, C.-K. Wu, S.-C. Chang, T.-M. Chen, and B.-W. Liou, Jpn. J. Appl. Phys. 44, 2516 (2005).

${ }^{5}$ G. B. Palmer, K. R. Poeppelmeier, and T. O. Mason, Chem. Mater. 9, 3121 (1997).

${ }^{6}$ P. Barquinha, G. Goncalves, L. Pereira, R. Martins, and E. Fortunato, Thin Solid Films 515, 8450 (2007).

${ }^{7}$ G. Gonçalves, E. Elangovan, P. Barquinha, L. Pereira, R. Martins, and E. Fortunato, Thin Solid Films 515, 8562 (2007).

${ }^{8}$ P. J. Cannard and R. J. D. Tilley, J. Solid State Chem. 73, 418 (1988).

${ }^{9}$ H. Ohta, W.-S. Seo, and K. Koumoto, J. Am. Ceram. Soc. 79, 2193 (1996).

${ }^{10}$ T. Sameshima, S. Usui, and H. Sekiya, IEEE Electron Device Lett. 7, 276 (1986).

${ }^{11}$ M. Nakata, K. Takechi, S. Yamaguchi, E. Tokumitsu, H. Yamaguchi, and S. Kaneko, Jpn. J. Appl. Phys. 48, 115505 (2009).

${ }^{12}$ M. P. Taylor, D. W. Readey, M. F. A. M. van Hest, C. W. Teplin, J. L. Alleman, M. S. Dabney, L. M. Gedvilas, B. M. Keyes, B. To, J. D. Perkins, and D. S. Ginley, Adv. Funct. Mater. 18, 3169 (2008).

${ }^{13}$ M. Nakata, K. Takechi, K. Azuma, E. Tokumitsu, H. Yamaguchi, and S. Kaneko, Appl. Phys. Express 2, 021102 (2009).
${ }^{14}$ H.-M. Kim, S.-K. Jung, J.-S. Ahn, Y.-J. Kang, and K.-C. Je, Jpn. J. Appl. Phys. 42, 223 (2003).

${ }^{15}$ P. Ch. van der Wilt, B. D. van Dijk, G. J. Bertens, R. Ishihara, and C. I. M. Beenakker, Appl. Phys. Lett. 79, 1819 (2001).

${ }^{16}$ R. Ishihara, P. Ch. van der Wilt, B. D. van Dijk, A. Burtsev, J. W. Metselaar, and C. I. M. Beenakker, Thin Solid Films 427, 77 (2003).

${ }^{17}$ M. He, Ph.D. dissertation, Delft University of Technology, The Netherlands, 2007.

${ }^{18}$ T. Ashida, A. Miyamura, Y. Sato, T. Yagi, N. Taketoshi, T. Baba, and Y. Shigesato, J. Vac. Sci. Technol. A 25, 1178 (2007).

${ }^{19}$ T. Szörényi, L. D. Laude, I. Bertóti, Z. Kántor, and Zs. Geretovszky, J. Appl. Phys. 78, 6211 (1995).

${ }^{20}$ M. Chen, X. Wang, Y. H. Yu, Z. L. Pei, X. D. Bai, C. Sun, R. F. Huang, and L. S. Wen, Appl. Surf. Sci. 158, 134 (2000).

${ }^{21}$ T. Aoki, T. Hatanaka, and D. C. Look, Appl. Phys. Lett. 76, 3257 (2000).

${ }^{22}$ Y. Hatanaka, M. Niraula, A. Nakamura, and T. Aoki, Appl. Surf. Sci. 175-176, 462 (2001).

${ }^{23}$ T. Minami, H. Sato, H. Nanto, and S. Takata, Jpn. J. Appl. Phys., Part 1 25, 776 (1986). 